\title{
Determination of dynamic relative permeability in ultra-low permeability sandstones via X-ray CT technique
}

\author{
Haiyong Zhang $\cdot$ Shunli He $\cdot$ Chunyan Jiao $\cdot$ \\ Guohua Luan $\cdot$ Shaoyuan Mo $\cdot$ Xuejing Guo
}

Received: 5 September 2013/Accepted: 6 January 2014 / Published online: 20 January 2014

(C) The Author(s) 2014. This article is published with open access at Springerlink.com

\begin{abstract}
Forced oil-water displacement is the crucial mechanisms of secondary oil recovery. The knowledge of relative permeability is required in the simulation of multiphase flow in porous media. Obvious dynamic effect of capillary pressure occurs in that the formation of ultra-low permeability reservoir (the permeability is $<1 \times$ $10^{-3} \mu \mathrm{m}^{2}$ ) is tight and the pores and throats are very small. In addition, the significant capillary end effect causes serious errors when calculating relative permeabilities. For these reasons, the JBN method (neglecting capillary pressure) does not apply. Therefore, the dynamic capillary pressure and capillary end effects should be taken into account. This work focuses on calculating two-phase relative permeability of ultra-low permeability reservoir through considering the dynamic capillary pressure and eliminating the influence of capillary end effects. Firstly, laboratory core scale measurements of in situ water phase saturation history based on X-ray CT scanning technique were used to estimate relative permeability. Secondly, a mathematical model of two-phase relative permeability considering the dynamic capillary pressure was established. The basic problem formulations, as well as the more specific equations, were given, and the results of
\end{abstract}

H. Zhang $(\bowtie) \cdot S$. He $\cdot$ S. Mo $\cdot$ X. Guo

School of Petroleum Engineering, China University

of Petroleum, Beijing, China

e-mail: zhy_1988@126.com

C. Jiao

Langfang Branch, CNPC Research Institute of Petroleum

Exploration and Development, Langfang, China

G. Luan

CNPC Petroleum Safety and Environmental Protection Institute of Technology, Beijing, China comparison using experimental data are presented and discussed. Results indicate that the dynamic capillary pressure measured at laboratory core scale in ultra-low permeability rocks has a significant influence on the estimation of unsteady-state relative permeability. The mathematical calculating method was compared with the history matching method and the results were close, suggesting reliability for ultra-low permeability reservoirs. Importantly, the proposed methods allow measurement of relative permeability from a single experiment. Potentially this represents a great time savings.

Keywords Dynamic capillary effect $\cdot$ X-ray CT . Ultra-low permeability $\cdot$ Relative permeability

\section{List of symbols}

$\alpha_{\mathrm{s}} \quad$ Empirical constant, which is equal to 0.1

$\varphi \quad$ Porosity (\%)

$\varphi_{\mathrm{CT}} \quad$ The CT-measured porosity (\%)

$\mu_{\mathrm{w}} \quad$ Viscosity of the wetting phase ( $\mathrm{mPa} \mathrm{s}$ )

$P_{\mathrm{e}} \& \lambda \quad$ Factors of capillary pressure-saturation relationships in the Brook-Corey model

$p_{\mathrm{c}}^{\mathrm{d}} \quad$ The dynamic capillary pressure (MPa)

$p_{\mathrm{c}}^{\mathrm{e}} \quad$ The steady-state capillary pressure (MPa)

$p_{\mathrm{o}} \quad$ The capillary pressure of oil phase (MPa)

$p_{\mathrm{w}} \quad$ The capillary pressure of water phase (MPa)

$\tau_{\mathrm{s}}$ or $\tau \quad$ The coefficient of dynamic capillary pressure $\left(\mathrm{kg} \mathrm{m}^{-1} \mathrm{~s}^{-1}\right)$

$\mathrm{K} \quad$ Absolute permeability $\left(10^{-3} \mu \mathrm{m}^{2}\right)$

$\rho_{\mathrm{w}} \quad$ Density of the wetting phase $\left(\mathrm{kg} \mathrm{m}^{-3}\right)$

$\mathrm{g} \quad$ Gravity acceleration $\left(\mathrm{m} \mathrm{s}^{-2}\right)$

L Characteristic length $(\mathrm{cm})$

D Diameter (cm)

$S_{\mathrm{oi}} \quad$ Initial oil saturation (\%) 
$S_{\text {or }} \quad$ Residual oil saturation (\%)

$S_{\mathrm{wc}} \quad$ Connate water saturation (\%)

$K_{\mathrm{rw}}\left(S_{\mathrm{or}}\right) \quad$ Relative permeability to water under residual oil saturation, dimensionless

$K_{\mathrm{ro}}\left(S_{\mathrm{wc}}\right) \quad$ Relative permeability to oil under connate water saturation, dimensionless

$K_{\text {ro }} \quad$ Relative permeability to oil, dimensionless

$K_{\mathrm{rw}} \quad$ Relative permeability to water, dimensionless

$u_{\mathrm{w}} \quad$ Flow velocity of the wetting phase $\left(\mathrm{cm} \mathrm{s}^{-1}\right)$

$u_{\mathrm{z}} \quad$ Injection velocity of the wetting phase at the inlet end of core $\left(\mathrm{cm} \mathrm{s}^{-1}\right)$

$K_{\text {air }} \quad$ Absolute permeability to air $\left(10^{-3} \mu \mathrm{m}^{2}\right)$

$K_{\text {wat }} \quad$ Absolute permeability to water $\left(10^{-3} \mu \mathrm{m}^{2}\right)$

EOR Oil recovery (\%)

$S_{\text {we }} \quad$ Water saturation at the core outlet (\%)

$S_{\mathrm{wi}} \quad$ Initial water saturation (\%)

$f_{\mathrm{o}} \quad$ Oil ratio at the core outlet $(\%)$

$f_{\mathrm{w}} \quad$ Water ratio at the core outlet (\%)

I Injection ability at some time/the initial injection ability

$\bar{V}_{\mathrm{o}}(t) \quad$ Dimensionless cumulative oil production volume

$\bar{V}(t) \quad$ Dimensionless liquid production volume

$\mathrm{CT}_{\text {Dry }} \quad$ The CT number for the dry core sample

$\mathrm{CT}_{\text {Air }}$ The CT number for air

$\mathrm{CT}_{\text {Phase1 }}$ The CT number for phase1

$\mathrm{CT}_{\text {Phase2 }}$ The CT number for phase2

$\mathrm{CT}_{\text {Saturated }}$ The CT number for the core saturated by one single phase

$\mathrm{CT}_{\text {Two }} \quad$ The CT number for two-phase liquids

$\mathrm{CT}_{\text {Grain }} \quad$ The $\mathrm{CT}$ number for the grain of core sample

$\mathrm{CT}_{\text {ow }} \quad$ The CT number for the oil-water two phases

$\mathrm{CT}_{\mathrm{w}} \quad$ The CT number for the water phase

$\mathrm{CT}_{\mathrm{O}} \quad$ The $\mathrm{CT}$ number for the oil phase

$\mathrm{CT}_{\text {or }} \quad$ The $\mathrm{CT}$ number for the core sample saturated by oil phase

\section{Introduction}

Simulation of multiphase flow in porous media requires relative permeability functions to make estimates of productivity, injectivity, and ultimate recovery from oil reservoirs for evaluation and planning of production operations (Honarpour and Mahmood 1988). Hence, measurements of relative permeability in the laboratory or theoretical models remain an important subject in reservoir modeling.

The laboratory methods used to calculate relative permeability functions include centrifuge, steady- and unsteady-state techniques. The centrifuge method has limitations including loss of information on the low saturation region that cannot be gained from the production data at low mobility ratio (Hirasaki et al. 1995) and the replacement of viscous forces with a range of centrifugal forces for unsteady-state displacement processes that are rate dependent was concerned (Ali 1997). Steady-state methods have disadvantages, especially in the case of low permeability rocks where it is laborious to reach multiple steady states, and capillary pressures and capillary end effects are significant (Firoozabadi and Aziz 1988; Kamath et al. 1995; Huang and Honarpour 1998). Additionally, the distribution of phases during the simultaneous injection of fluids through the core may not represent the actual displacement process in the reservoir.

The unsteady-state method is much less time consuming than the steady-state method and is more representative of the reservoir flow mechanisms, but the mathematical analysis of the unsteady-state procedure is more difficult and the capillary pressure has a significant effect on saturation distribution and recovery, capillary forces dominate multiphase flow in low-permeability rocks. Akin and Kovscek (1999) indicated that the (unsteady) JBN technique neglecting the capillary forces could lead to inaccurate assessment of relative permeability in some cases. Therefore, most conventional unsteady techniques do not apply.

There are also methods that infer relative permeability by history matching observable parameters such as pressure drop and saturation profile history. The relative permeability curves are adjusted until a good match is obtained with the experimental data. The limitation is that the description of the actual shape of the relative permeability curves can cause errors (Hirasaki et al. 1995).

This work focuses on calculating two-phase dynamic relative permeability of ultra-low permeability reservoir. Firstly, the dynamic capillary pressure was investigated through displacement tests and conventional mercury injection tests. Secondly, the conventional JBN technique was used to calculate two-phase relative permeability. Results indicated that the JBN method did not apply. Thirdly, laboratory core scale measurements of in situ water phase saturation history via X-ray CT technique were used to estimate relative permeability through history matching method. Finally, a mathematical model of twophase relative permeability considering the dynamic capillary pressure was established. The basic problem formulations, as well as the more specific equations, were given and the results of comparison with experiments are presented and discussed.

\section{Dynamic capillary pressure}

Dynamic capillary pressure refers to capillary pressure relating to variation of wetting phase saturation as a 


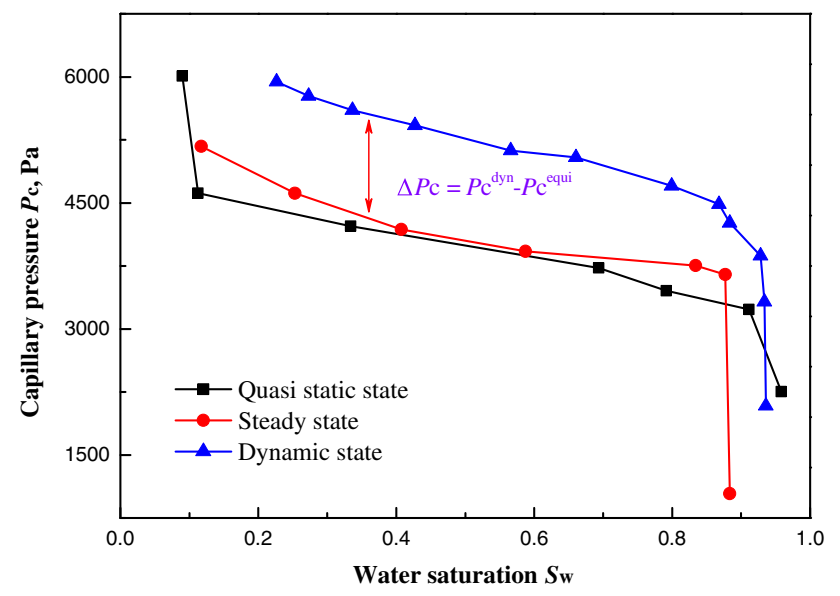

Fig. 1 The dynamic effect of capillary pressure (Topp et al. 1967)

function of time when the oil-water interface fails to reach an equilibrium state. Topp et al. (1967) found the dynamic effect of capillary pressure (Fig. 1) and the dynamic capillary pressure is larger than the static capillary pressure under the same water saturation at larger displacing velocity.

Stauffer (1978) studied the dynamic effect of capillary pressure by displacement experiment and revealed that the capillary pressure-saturation relationship is not unique. The dynamic capillary pressure is larger than quasi-static capillary pressure. He also established empirical equations between capillary pressure and saturation (Eqs. 1, 2).

$p_{\mathrm{c}}^{\mathrm{d}}-p_{\mathrm{c}}^{\mathrm{e}}\left(S_{\mathrm{w}}\right)=-\tau_{\mathrm{s}}\left(\partial S_{\mathrm{w}} / \partial t\right)$

$\tau_{\mathrm{s}}=\frac{\alpha_{\mathrm{s}} \mu_{\mathrm{w}} \varphi}{K \lambda}\left(\frac{P_{\mathrm{e}}}{\rho_{\mathrm{w}} g}\right)^{2}$

Hassanizadeh and Gray (1990) confirmed the validity of equations proposed by Stauffer (1978). Wildenschild et al. (2001) found that the dynamic capillary pressure is obvious for sands (soil samples) and larger capillary pressure occurs at faster displacing speed. While there is no dynamic effect of capillary pressure for fine-grained sands (soil samples).

Previous studies demonstrate the existence of dynamic capillary pressure in the majority of sands or soils, but there is a lack of study about the dynamic capillary pressure in ultra-low permeability sandstone. Thus, waterflooding tests and mercury injection tests were conducted to analyze whether dynamic capillary pressure exists in ultralow permeability sandstone.

Waterflooding test

\section{Experimental data and procedures}

To analyze the capillary pressure, we measured the flow velocity under different conditions of pressure differential.
The parameters of cores from Changqing oil field in Ordos Basin can be seen in Table 1. The experimental fluids are standard brine and kerosene (Table 2). In this case, kerosene is the non-wetting phase. Figure 2 is the experimental apparatus and the displacement processes were repeated six times for six core samples. The results of two core samples were showed in Fig. 3.

The waterflooding procedures are given as follows (SY/ T 5345-2007; Donaldson and Dean 1966):

1. Measure the absolute permeability of core at the specific back pressure (1,000 psi).

2. Saturate the core with oil. Under the condition of constant pressure differential, close the 'Pneumatic Valve' of oil phase (1-2 in Fig. 2) and then open the 'Pneumatic Valve' of water phase (1-1 in Fig. 2) to inject water into the core under the condition of one and the same pressure gradient.

3. Record the accumulative oil volume at the outlet end of core, and then calculate the flow velocity.

4. Measure the accumulative oil volume under the condition of a second and the same pressure gradient until the whole waterflooding tests are conducted.

Finally, we obtain the seepage curves of waterflooding (Fig. 3).

\section{Results and analysis}

The waterflooding curves (Fig. 3) of the flow velocity as a function of pressure gradient are nonlinear proving the existence of additional seepage resistance. Since the pores and throats of ultra-low permeability sandstone are very small, this additional seepage resistance is most likely capillary force. Hence, a further study was done through conventional mercury injection experiments.

\section{Conventional mercury injection test}

We analyzed the capillary pressure under the conditions of different injection velocities by conventional mercury injection tests with four cores of similar physical properties as the previous waterflooding tests.

The procedures of mercury injection test are as follows:

1. Wash oil and then dry off it for the first core sample.

2. Measure the parameters of core including permeability, porosity, density and the volume $\left(V_{f}\right)$.

3. Inject mercury into the core at an initial pressure of $0.3 \mathrm{MPa} .10 \mathrm{~s}$ later, record the pressure value $\left(P_{\mathrm{c}}\right)$ and the injected mercury volume $\left(V_{\mathrm{Hg}}\right)$. Repeat this process every $10 \mathrm{~s}$ by increasing the injection pressure about 0.4-0.5 MPa every time until the mercury injection test is completed. It means that the mercury injection time for the first core sample is $10 \mathrm{~s}$. 
Table 1 The parameters of cores used in the water flooding tests

\begin{tabular}{lllllllll}
\hline Sample & $\mathrm{L}(\mathrm{cm})$ & $\mathrm{D}(\mathrm{cm})$ & $\mathrm{K}\left(10^{-3} \mu \mathrm{m}^{2}\right)$ & $\varphi(\%)$ & Dry weight $(\mathrm{g})$ & Wet weight $(\mathrm{g})$ & Saturated volume $(\mathrm{ml})$ & $S_{\text {oi }}(\%)$ \\
\hline $1 \#$ & 16.85 & 9.90 & 0.14 & 10.8 & $3,205.4$ & $3,294.1$ & 111.15 & 79.39 \\
$2 \#$ & 19.65 & 9.85 & 0.73 & 11.7 & $3,525.7$ & $3,649.6$ & 155.36 & 88.73 \\
\hline
\end{tabular}

Table 2 The parameters of the testing fluids

\begin{tabular}{lll}
\hline Fluid & Viscosity $(\mathrm{mPa} \mathrm{s})$ & Density $\left(\mathrm{g} \mathrm{m}^{-3}\right)$ \\
\hline Standard brine & 1.35 & 1.050 \\
Kerosene & 1.53 & 0.772 \\
\hline
\end{tabular}

4. Calculate the mercury saturation $\left(S_{\mathrm{Hg}}\right)$ through the following equation,

$S_{\mathrm{Hg}}=V_{\mathrm{Hg}} /\left(\varphi V_{f}\right)$

5. The mercury injection time for the other three core samples is 60,300 and $600 \mathrm{~s}$, respectively. Repeat the four procedures from step 1 to 4 and then, the capillary pressure-mercury saturation relationships can be obtained in Fig. 4.

In Fig. 4, we see that the capillary pressure becomes larger at a higher mercury injection velocity caused by higher injection pressure. The capillary pressure differs not quite when the mercury injection time is 300 and $600 \mathrm{~s}$, indicating it can be approximately regarded as static capillary pressure when the mercury injection time reaches to $300 \mathrm{~s}$ (namely, the system reaches an equilibrium state).

We calculate the coefficient $\tau_{\mathrm{s}}$ of ultra-low permeability reservoir using Eq. 2, they can reach to $10^{11}$ $10^{13} \mathrm{~kg} \mathrm{~m}^{-1} \mathrm{~s}^{-1}$ (Table 3) while the coefficients $\tau_{\mathrm{s}}$ calculated by predecessors (Stauffer 1978; Wanna 1982; Hassanizadeh and Gray 1990) are only $10^{4}-$ $10^{7} \mathrm{~kg} \mathrm{~m}^{-1} \mathrm{~s}^{-1}$. Mirzaei and Das (2007) found that there is a significant variation on the reported values of $\tau_{\mathrm{s}}$ in the literatures $\left(0-10^{9} \mathrm{~kg} \mathrm{~m}^{-1} \mathrm{~s}^{-1}\right)$ depending on the size $\left(10^{-3}-1 \mathrm{~m}\right)$ and geometry (2D or $\left.3 \mathrm{D}\right)$ of domains. The dynamic coefficient values up to $10^{11} \mathrm{~kg} \mathrm{~m}^{-1} \mathrm{~s}^{-1}$ were found by Das et al. (2007) in their experiments on fine sands with the permeability about $3 \times 10^{-10} \mathrm{~m}^{2}$, which is much larger than that of ultralow permeability core sample $\left(<1 \times 10^{-3} \mu \mathrm{m}^{2}\right.$ or $\left.1 \times 10^{-15} \mathrm{~m}^{2}\right)$. This indicates that the coefficients $\tau_{\mathrm{s}}$ are very big in ultra-low permeability reservoir leading to obvious dynamic effect of capillary pressure. The tighter the reservoir formation is, the larger the coefficient $\tau_{\mathrm{s}}$ will be. This result is in accordance with the result of Das and Mirzaei (2012) where $\tau$ is also found to increase in the regions of less permeability.
Two-phase flow model

Then a two-phase flow model considering the dynamic capillary pressure was established to analyze the influence of dynamic capillary pressure on water saturation distribution.

The dynamic capillary pressure is given by

$p_{\mathrm{o}}-p_{\mathrm{w}}=p_{\mathrm{c}}^{\mathrm{d}}=p_{\mathrm{c}}^{\mathrm{e}}-\tau\left(\partial S_{\mathrm{w}} / \partial t\right)$

The basic differential equation of each phase is given as follows. For the oil phase,

$\frac{\partial}{\partial x}\left(\frac{K K_{\mathrm{ro}}}{\mu_{\mathrm{o}}} \frac{\partial p_{\mathrm{o}}}{\partial x}\right)=\varphi \frac{\partial S_{\mathrm{o}}}{\partial t}$

For the water phase,

$\frac{\partial}{\partial x}\left(\frac{K K_{\mathrm{rw}}}{\mu_{\mathrm{w}}} \frac{\partial p_{\mathrm{w}}}{\partial x}\right)=\varphi \frac{\partial S_{\mathrm{w}}}{\partial t}$

Thus substituting Eq. 5 into 6 and then combining with 4, the two-phase flow model is given by

$\frac{\partial}{\partial x}\left(\frac{K K_{\mathrm{ro}}}{\mu_{\mathrm{o}}} \frac{\partial\left(p_{\mathrm{w}}+p_{\mathrm{c}}^{\mathrm{e}}-\tau\left(\partial S_{\mathrm{w}} / \partial t\right)\right)}{\partial x}\right)+\frac{\partial}{\partial x}\left(\frac{K K_{\mathrm{rw}}}{\mu_{\mathrm{w}}} \frac{\partial p_{\mathrm{w}}}{\partial x}\right)=0$

The solution of the two-phase flow model was obtained through the numerical method of implicit pressure-explicit saturation (IMPES). The results can be seen in Fig. 5, indicating that a reduction of forwarding velocity of water saturation is induced considering the dynamic capillary pressure and the existence of dynamic capillary pressure has a significantly influence on the water saturation distribution resulting in the smaller saturation gradient and lower oil production.

\section{Conventional methodology}

Unsteady-state techniques

Laboratory equipment (Fig. 2) and procedures are available (see the previous section of waterflooding test) for making the unsteady-state measurements under simulated reservoir condition (SY/T 5345-2007; Donaldson and Dean 1966). Table 2 is the parameters of fluids. The salinity of standard brine is $80 \mathrm{~g} / \mathrm{l}$, where the ratio of $\mathrm{NaCl}: \mathrm{CaCl}_{2}$ : 
Fig. 2 The flowchart of AFS300 device. 1 pneumatic valve, 2 bypass and test, 3 ISCO pump, 4 back-pressure valve, 5 standard brine, 6 kerosene, 7 low DP (0-8 Psi), 8 Med DP (8-125 Psi), 9 high DP (125-300 Psi), 10 upstream pressure sensor, 11 downstream pressure sensor, 12 confining pressure sensor, 13 triaxial coreholder
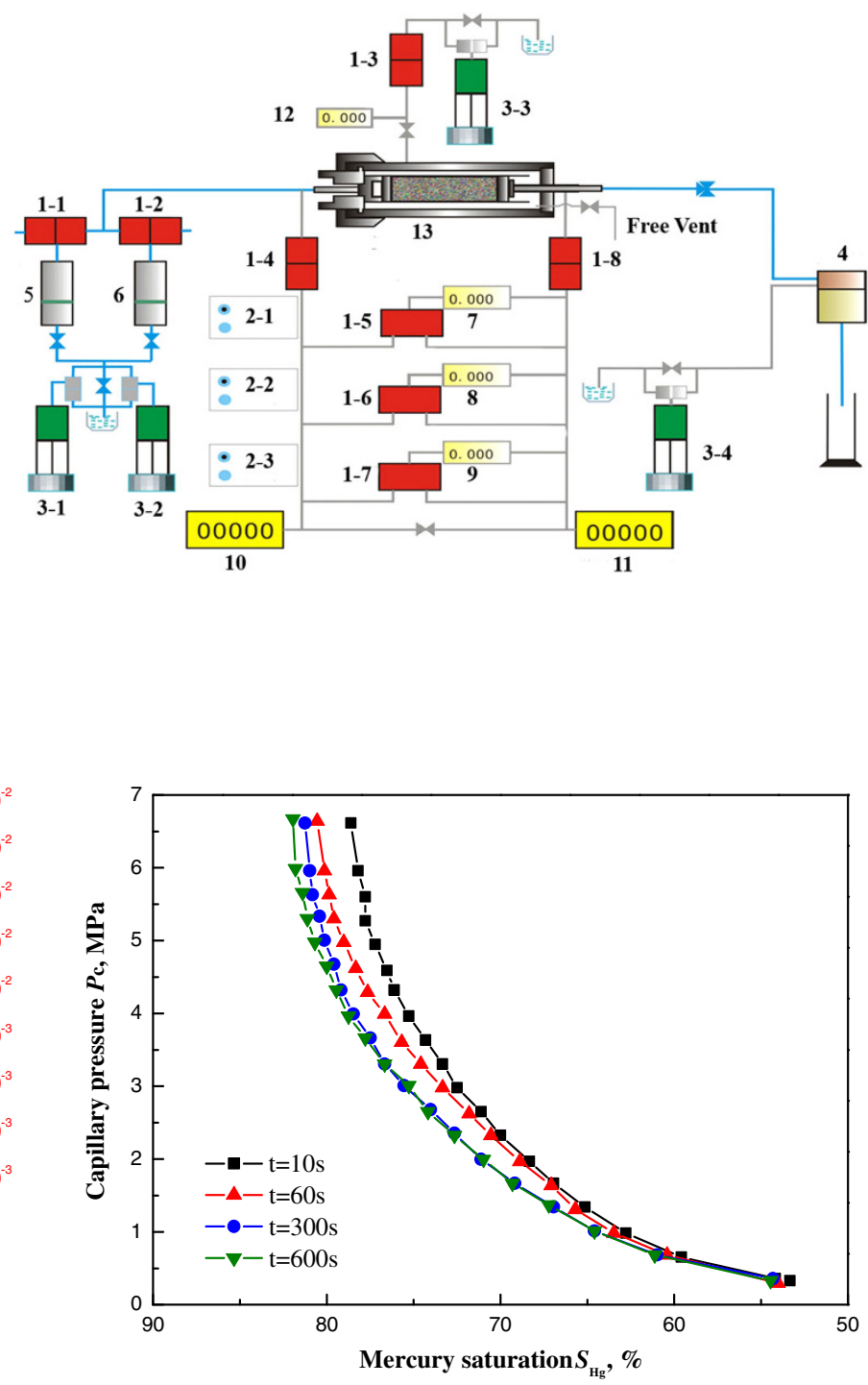

Fig. 4 The capillary pressure curves of different mercury injection rates

permeability rocks, mainly manifested in the determination of outlet end oil production, especially when the oil ratio is very low, due to the capillary end effects (Qadeer et al. 1988; Huang and Honarpour 1998).

The flow of oil and water is quite complex due to the very small pores and throats of ultra-low permeability sandstone. Thus, it is difficult to master the time interval when recording the output data at the outlet end of core sample. The capillary end effect is obvious in a small time interval resulting in large errors of production data, while the variable features of production index cannot be reflected in a large time interval and hence, a modification of production data is requested following these principles. Firstly, keep the connate water saturation and residual oil saturation in their original values. Secondly, a smoothing process is needed if the curve is not smooth. Finally, just

Laboratory tests have indicated that the measurements of relative permeability are far from easy for ultra-low

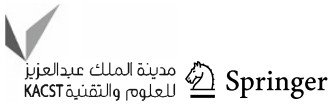


Table 3 The parameters of core samples used for the calculation of coefficient $\left(\tau_{\mathrm{s}}\right)$

\begin{tabular}{llllllllll}
\hline Sample & $\lambda$ & $P_{\mathrm{e}}(\mathrm{MPa})$ & $\alpha$ & $\mu_{\mathrm{w}}(\mathrm{mPa} \mathrm{s})$ & $\varphi(\%)$ & $\mathrm{K}\left(10^{-3} \mu \mathrm{m}^{2}\right)$ & $\rho_{\mathrm{w}}\left(\mathrm{kg} \mathrm{m}^{-3}\right)$ & ${\mathrm{g}\left(\mathrm{m} \mathrm{s}^{-2}\right)}_{\tau_{\mathrm{s}}\left(\mathrm{kg} \mathrm{m}^{-1} \mathrm{~s}^{-1}\right)}$ \\
\hline $3 \#$ & 0.859 & 0.131 & 0.1 & 1.35 & 9.77 & 0.033 & 1,050 & 9.8 & $7.54 \mathrm{E}+13$ \\
$4 \#$ & 0.775 & 0.049 & 0.1 & 1.35 & 12.65 & 0.039 & 1,050 & 9.8 & $1.28 \mathrm{E}+13$ \\
$5 \#$ & 1.046 & 0.04 & 0.1 & 1.35 & 12.01 & 0.057 & 1,050 & 9.8 & $4.11 \mathrm{E}+12$ \\
$6 \#$ & 0.374 & 0.009 & 0.1 & 1.35 & 12 & 0.1 & 1,050 & 9.8 & $3.31 \mathrm{E}+11$ \\
$7 \#$ & 0.637 & 0.022 & 0.1 & 1.35 & 14.2 & 0.122 & 1,050 & 9.8 & $1.13 \mathrm{E}+12$ \\
$8 \#$ & 0.568 & 0.031 & 0.1 & 1.35 & 12.27 & 0.083 & 1,050 & 9.8 & $3.19 \mathrm{E}+12$ \\
\hline
\end{tabular}

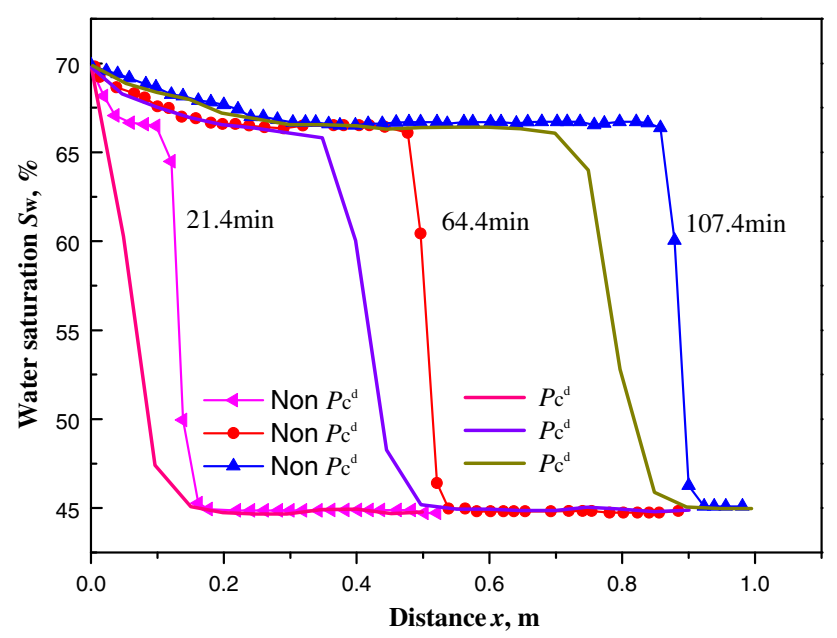

Fig. 5 The water saturation profiles along the simulated length at different times without and with dynamic capillary pressure

modify the oil production data in that the liquid production data are reliable at each stage.

\section{Results}

Take core 9\# for example, Fig. 6a shows the difference of original and corrected oil production, the modified relative permeability curves are obtained (Fig. 6b) by substituting the corrected oil ratio $f_{\mathrm{o}}$ into the equations of JBN method. The relative permeability curves using revised data are almost two intersecting straight lines. Since straight relative permeability curves represent the percolation in reservoirs without capillary forces, it is not a good representation of relative permeability for ultra-low permeability reservoir with obvious capillary forces, indicating that the JBN method does not apply.

\section{Proposed methodology}

\section{X-ray CT technique}

According to previous analysis, new methods are needed for calculating relative permeability curves. The errors of oil production data caused by the capillary end effect may play a major role in the feature of relative permeability appearing in Fig. 6b. To eliminate the influence of capillary end effect, X-ray CT technique was used to obtain saturation profiles as a function of time during the unsteady-state oil-water displacement process.

\section{Apparatus and principles}

A LightSpeed 8 CT Scanner from GE was used for in situ saturation measurements. For imaging, the voxel dimension is $0.25 \times 0.25 \times 0.625 \mathrm{~mm}$, the scanning time frequency is $0.5 \mathrm{~s}$ and the image reconstruction time is $0.167 \mathrm{~s}$. The scanning data are processed by an image processing software (CTIAS 1.0, developed by RIPED) that comes with the X-ray CT scanning system.

The main principles of X-ray CT scanning for core analysis have been expressed in several early papers which span both core description and coreflooding (Ayala and Aziz 1993; Hughes et al. 1996). Vinegar and Wellington (1987) provided the industry with a comprehensive study on the methodology and application of CT scanning for coreflood monitoring. Withjack (1988) demonstrated CT scanning for special core analysis including CT porosity determination and relative permeability measurement. The fundamental principles of the X-ray CT technique are given as follows. The scanning data is converted by high speed computer and then the images can be displayed on the screen or photographed. The reconstructed images are usually expressed in CT values. Since different materials have different CT values, the CT values are used to separate the phases. We can take advantage of the different CT values along the core length to identify the fluid saturation.

A single scan provides every position of saturation at a specific time. Image processing of the CT data provides values of water saturation (fraction of the pore volume occupied by water) along the core length. The resolution of images processed by the image processing software is $512 \times 512$ pixel. By dividing the density difference of each imaging unit inside a core into 256 gray scales, the pore structure features are reflected, and then the density, 
Table 4 The parameters of cores and the testing results

\begin{tabular}{lllllllllll}
\hline Samples & $\mathrm{L}(\mathrm{cm})$ & $\mathrm{D}(\mathrm{cm})$ & $\mathrm{K}\left(10^{-3} \mu \mathrm{m}^{2}\right)$ & $\varphi(\%)$ & $S_{\text {or }}(\%)$ & $K_{\mathrm{rw}}\left(S_{\mathrm{or}}\right)\left(10^{-3} \mu \mathrm{m}^{2}\right)$ & $S_{\mathrm{wc}}(\%)$ & $K_{\mathrm{ro}}\left(S_{\mathrm{wc}}\right)\left(10^{-3} \mu \mathrm{m}^{2}\right)$ & $\mathrm{EOR}(\%)$ \\
\hline $9 \#$ & 20.29 & 9.93 & 0.69 & 10.2 & 34.54 & 0.055 & 55.74 & 0.192 & 21.95 \\
$10 \#$ & 19.87 & 9.84 & 4.70 & 13.85 & 24.55 & 0.403 & 63.73 & 2.919 & 32.30 \\
$11 \#$ & 18.35 & 9.88 & 1.08 & 5.9 & 43.01 & 0.044 & 48.53 & 0.402 & 16.43 \\
\hline
\end{tabular}

porosity and saturation of core can be obtained through CT number.

Here are the specific principles for calculating the water saturation profiles via X-ray $\mathrm{CT}$ technique. The $\mathrm{CT}$ number for dry core sample, $\mathrm{CT}_{\text {Dry }}$, can be calculated by

$\mathrm{CT}_{\text {Dry }}=(1-\varphi) \mathrm{CT}_{\text {Grain }}+\varphi \mathrm{CT}_{\text {Air }}$

The CT number for a core sample saturated with onephase liquid (Phase1 or Phase2), $\mathrm{CT}_{\text {Saturated, }}$ can be calculated by

$\mathrm{CT}_{\text {Satured } 1}=(1-\varphi) \mathrm{CT}_{\text {Grain }}+\varphi \mathrm{CT}_{\text {Phase } 1}$

$\mathrm{CT}_{\text {Satured2 }}=(1-\varphi) \mathrm{CT}_{\text {Grain }}+\varphi \mathrm{CT}_{\text {Phase2 }}$

Then the core porosity can be obtained (Akin and Kovscek 1999; Akin et al. 2000) based on Eqs. 11 and 12,

$\varphi=\frac{\mathrm{CT}_{\text {Satured1 }}-\mathrm{CT}_{\text {Dry }}}{\mathrm{CT}_{\text {Phase } 1}-\mathrm{CT}_{\text {Air }}}$

When two-phase liquids exist in the core, the CT number for two-phase liquids, $\mathrm{CT}_{\mathrm{Two}}$, can be calculated by

$\mathrm{CT}_{\text {Two }}=(1-\varphi) \mathrm{CT}_{\text {Grain }}+\varphi S_{\text {phase } 1} \mathrm{CT}_{\text {Phasel }}$

$$
+\varphi\left(1-S_{\text {phase1 } 1}\right) \mathrm{CT}_{\text {Phase2 }}
$$

Substituting Eq. 13 into 15 to replace $(1-\varphi) \mathrm{CT}_{\text {Grain }}$, the saturation of Phase 1 can be obtained

$S_{\text {Phase1 }}=\frac{1}{\varphi} \times \frac{\mathrm{CT}_{\text {Two }}-\mathrm{CT}_{\text {Satured2 }}}{\mathrm{CT}_{\text {Phase1 }}-\mathrm{CT}_{\text {Phase2 }}}$

In this work, Phase 1 is the water phase, Phase 2 is the oil phase and hence, the equation for calculating water saturation is given by

$S_{\mathrm{w}}=\frac{1}{\varphi} \times \frac{\mathrm{CT}_{\mathrm{ow}}-\mathrm{CT}_{\mathrm{or}}}{\mathrm{CT}_{\mathrm{w}}-\mathrm{CT}_{\mathrm{o}}}$

After the CT value of each position along the entire core length in the process of waterflooding is obtained and then, through the Eqs. 11-17, the water saturation profiles can be calculated. The particular image processing algorithms have been discussed by Akin et al. (2000). Further information about the experimental equipment, procedure, pure fluid CT numbers, and data processing is given by Akin and Kovscek (1999) and Akin et al. (2000).

The conditions of CT scanning: voltage $120 \mathrm{kV}$, electric current $100 \mathrm{~mA}$ and temperature $24{ }^{\circ} \mathrm{C}$. Water flooding tests were conducted under the condition of constant flow velocity, then the pressure differential and saturation
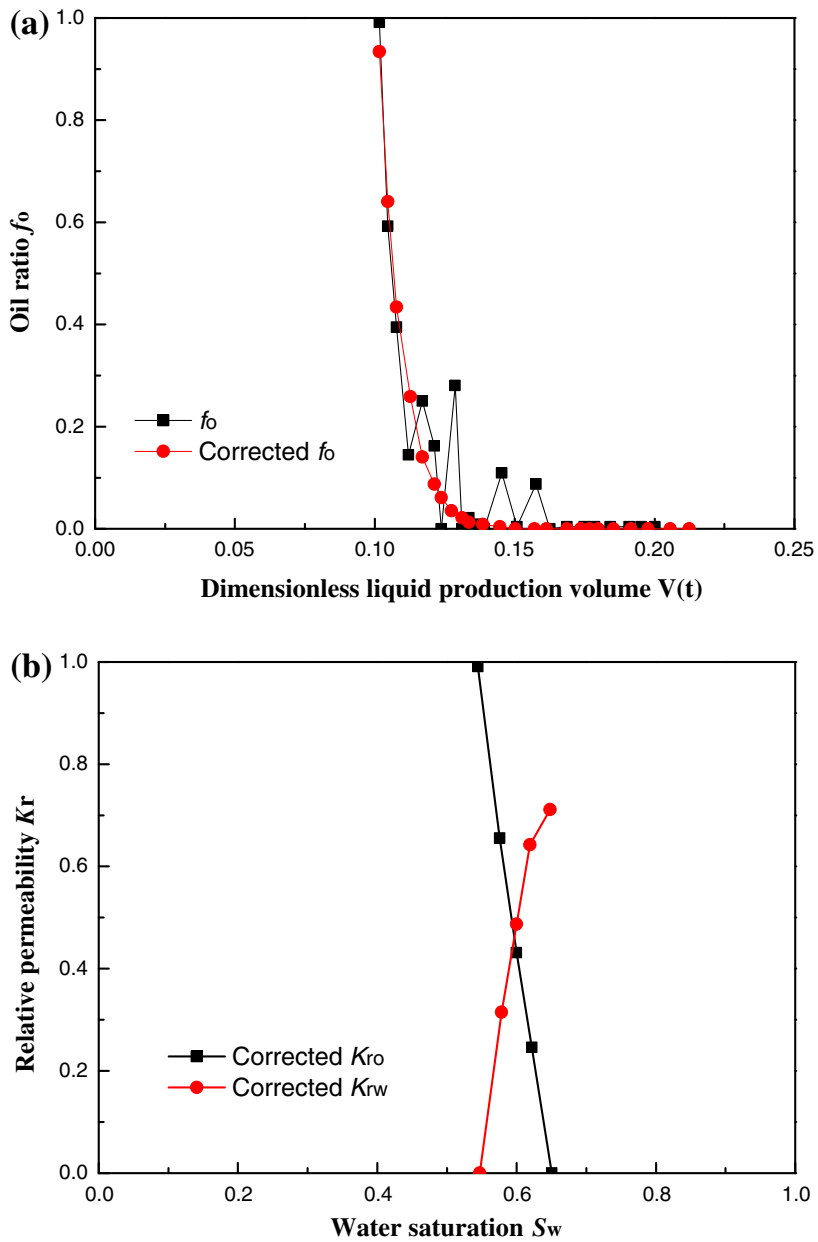

Fig. 6 The relative permeability calculated by the JBN method (a). The original and modified oil production data (b). The relative permeability curve from modified data

profile as a function of time along the entire core length could be determined. Figure 7 is the X-ray CT device structure (Hove et al. 1987).

\section{Experimental samples and fluids}

The core samples used in the tests were obtained from the Changqing oil reservoir in Ordos Basin. The samples were from outcrop. We characterized the rock with scanning electron microscopy analysis, cast thin section analysis and mercury intrusion analysis to analyze the microscopic pore structures. The parameters of experimental fluids (standard 
Fig. 7 X-ray CT device structure diagram

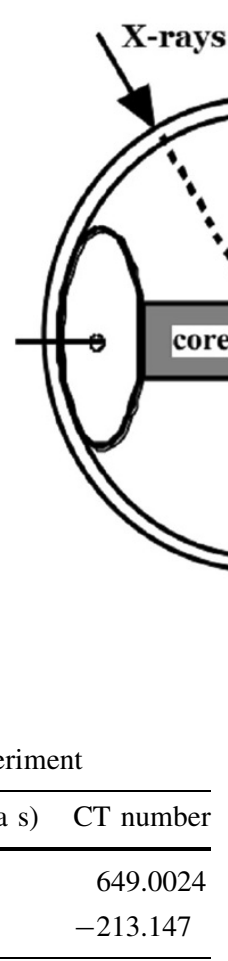

Table 5 The properties of fluids in X-ray CT experiment

\begin{tabular}{llll}
\hline Fluid & Density $\left(\mathrm{g} \mathrm{m}^{-3}\right)$ & Viscosity $(\mathrm{mPa} \mathrm{s})$ & CT number \\
\hline Standard brine & 1.055 & 1.2797 & 649.0024 \\
Kerosene & 0.8 & 1.25 & -213.147 \\
\hline
\end{tabular}

Table 6 The parameters of cores used in X-ray CT experiment

\begin{tabular}{llllll}
\hline Samples & $\mathrm{L}(\mathrm{cm})$ & $\mathrm{D}(\mathrm{cm})$ & $K_{\text {air }}\left(10^{-3} \mu \mathrm{m}^{2}\right)$ & $\varphi(\%)$ & $\varphi_{\mathrm{CT}}(\%)$ \\
\hline $12 \#$ & 17.5 & 2.390 & 0.4261 & 11.61 & 11.42 \\
$13 \#$ & 14.0 & 2.374 & 0.5010 & 13.22 & 13.13 \\
$14 \#$ & 15.0 & 2.400 & 0.4339 & 12.52 & 12.26 \\
\hline
\end{tabular}

brine and kerosene) and core samples used in X-ray CT tests can be seen in Tables 5 and 6 . The results show that the grain-size distributions of sandstones primarily consist of fine-medium size and very fine size. A majority of rock debris is moderately sorted. The mineral content mainly contains quartz and feldspar. The modes of contact between grains contain mostly lineal contact, then linealconcave and convex contact. The cementation types are dominated by basement-pore cementation and pore cementation. The cementing material of the Chang6 reservoir formation is mainly composed of rim growing chlorite. The average pore radius is $25-30 \mu \mathrm{m}$ while the average throat radius is $3-5 \mu \mathrm{m}$, indicating the throat size is much smaller than the pore size.

\section{Experimental procedures}

The core was saturated with kerosene in the initial condition and then standard brine was injected at a constant rate of $0.005 \mathrm{ml} / \mathrm{min}$ when the test began. The entire length of core was scanned along the flow direction through X-ray
CT during water flooding process. The essential procedures consist of the following steps:

1. Measure the porosity and permeability of cores.

2. Measure the CT-number data of fluids and the dry core samples.

3. Extract air for each core sample to reach a vacuum state and then saturate the core with one-phase fluid to measure the CT number for the core saturated with one-phase fluid (oil or water).

4. Calculate the porosity by Eq. 14 to obtain the porosity distribution profiles of each scanning plane both horizontal and vertical

5. Set the confining pressure to 2,000 Psi and the back pressure to $1,000 \mathrm{Psi}$, and then inject the standard brine into the core at a constant rate of $0.005 \mathrm{ml} / \mathrm{min}$. Meanwhile, measure the pressure differential at the inlet-outlet ends of core. Scanning is performed every $40 \mathrm{~min}$ for the early stage and then every $60 \mathrm{~min}$ before there is a water breakthrough at the outlet end of core. Afterwards the interval between images becomes longer, every $100 \mathrm{~min}$, since the change in saturations become slower with time.

6. Measure the volume of oil phase when the saturation distribution profiles no longer change.

7. Increase the displacing velocity to $0.01 \mathrm{ml} / \mathrm{min}$ and scan the core every $60 \mathrm{~min}$ to observe if there is oil produced at the outlet end of core. Finish the test when there is no increase of oil phase or there is no change of saturation.

\section{Results}

X-ray CT technique has been used by numerous scholars (Withjack 1988; Akin and Kovscek 1999; Akin et al. 2000; 
Lü et al. 2012). The CT-measured porosities were compared with the porosities measured by laboratory core analysis (Table 6). Since only the porosity of connected pores and throats was counted by CT technique and liquids could not flow into some very small pores and throats, the CT-measured porosities were a little smaller than the porosities measured by laboratory core analysis. For these reasons, the reliability of data obtained by X-ray CT technique is ensured.

Figure 8 shows the X-ray CT scanning data of dry cores 12\#, 13\# and 14\#. The raw CT-number data are converted to a map of porosity and then the water saturation profiles are obtained from subtracting images of raw CT data. In Figs. 9 and 11 , the water saturation distribution profiles along the core length of core 12\# are showed and the shape of water saturation profiles indicates relatively steep and sharp fronts in simulated reservoir conditions. As the increase of injected water at the inlet end of core, the common seepage area of oil-water two phases becomes very narrow and a pistonlike forwarding appears for the water front.

The microscopic pore structure, especially the sorting behavior, may play a major role in the piston-like movement. In the pore-scale coreflooding, piston-like movement is commonly observed experimentally. In this work, pistonlike movement was observed in two of the three samples via $C$ T scanning, while the third one was observed unpiston-like movement. We measured the pore structures of cores from the corresponding same positions where the CT scanning core samples were coring and found that the two core samples observed piston-like movement both have well-sorting behavior, while the sorting behavior of the core sample observed unpiston-like movement is poor. In addition, Fenwick and Blunt (1998) reported that there are two types of displacement (snap-off and piston-like invasion). For a given throat, piston-like invasion has a higher capillary pressure than snap-off, and so is favored, but can only occur if the adjacent pore or throat is already completely filled with wetting phase. Lenormand et al. (1988) obtained the same conclusions. Dixit et al. $(1998,2000)$ reported that the actual pore-scale mechanisms of snap-off and piston-like displacement depending on their contact angle. These may also be the reasons for the experimental phenomenon of piston-like movement.

\section{History matching (HM) method}

In the implementation of the optimization algorithm, the following objective function is proposed to be minimized for the verification of match between core flood reservoir model performance and predicted data to obtain two-phase relative permeability function. Thus, the difference between the position measured, $x^{\text {meas }}$, and the position calculated, $x^{\text {calc }}$, for each in situ saturation value as a

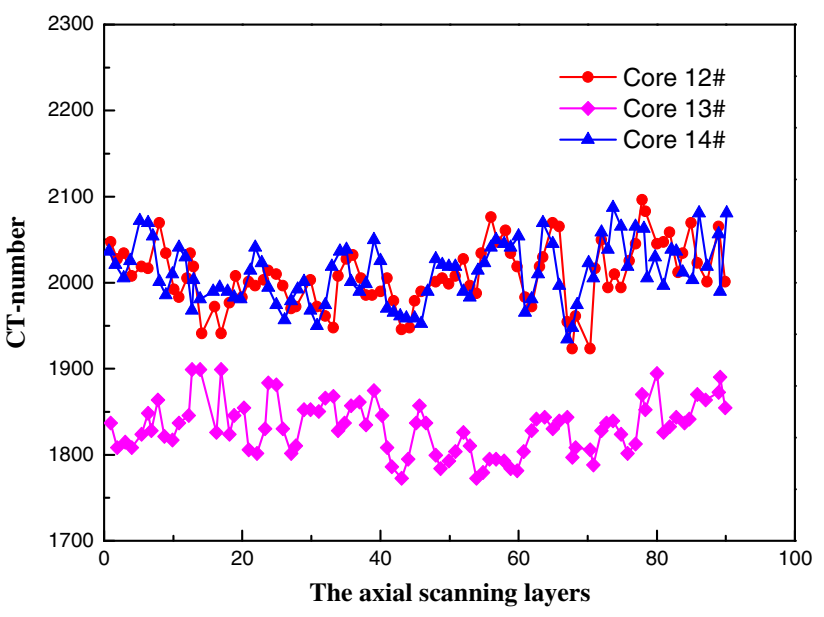

Fig. 8 CT-number data of experimental cores before saturated
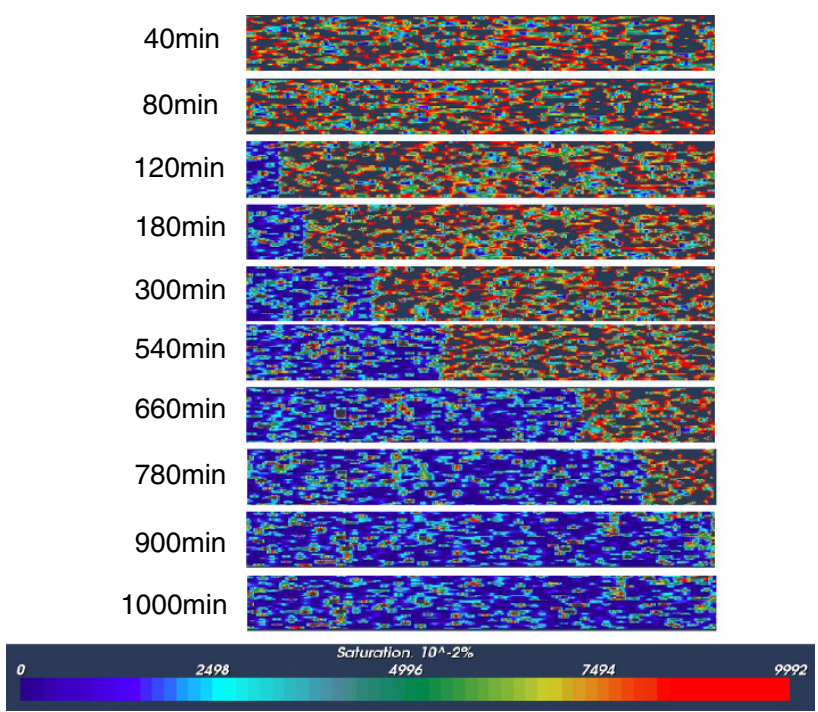

Fig. 9 The water saturation profiles along the core length at different times for core $12 \#$

function of time (Bail and Marsden 1956), is minimized. The objective function, $E$, is written

$E=\frac{\left[\sum_{i=0}^{N_{\text {prof }}} \sum_{j=1}^{N_{\text {sat }}}\left(x^{\text {calc }}\left(S_{w j}, t_{i}\right)-x^{\text {meas }}\left(S_{w j}, t_{i}\right)\right)^{2}\right]^{1 / 2}}{N_{\text {sat }} N_{\text {prof }}}$

where the product $N_{\mathrm{sat}} N_{\text {prof }}$ is the number of measured aqueous phase saturations to be matched. $E$ is minimized using simulated annealing algorithm (Kirkpatrick et al. 1983).

The power law model is used for the representation of relative permeability curves (Lake 1989),

$$
\begin{aligned}
& K_{\mathrm{ro}}=a\left(1-S_{\mathrm{we}}\right)^{n_{\mathrm{o}}} \\
& K_{\mathrm{rw}}=b S_{\mathrm{we}}^{n_{\mathrm{w}}} \\
& S_{\mathrm{we}}=\left(S_{\mathrm{w}}-S_{\mathrm{wi}}\right) /\left(1-S_{\mathrm{or}}-S_{\mathrm{wi}}\right)
\end{aligned}
$$


where $a, b, n_{\mathrm{o}}, n_{\mathrm{w}}$ are the parameters to be determined.

An efficient optimization algorithm (the simulated annealing algorithm) is implemented to adjust the parameters controlling relative permeability curves. The objective function $(E)$, Eq. 18, is then calculated by means of the numerical solution of the two-phase flow model (Janos et al. 2001) considering the dynamic capillary pressure (Eq. 7). Ucan et al. (1993) and Mitlin et al. (1998) have given the minimum analysis of the objective function using simulated annealing algorithm.

The relative permeability curves obtained were used to simulate the water saturation profiles versus time and the pressure differential versus time (Figs. 11, 12). The average relative error of the pressure differential data and the water saturation data is 12.5 and $8.1 \%$, respectively. The calculated history reproduced the experimental behavior well. The dynamic capillary pressure is considered in the percolation model during the history matching process. The larger value of $K_{\mathrm{rw}}$ at low water saturation affects the saturation front slightly at later times. The largest water saturations will affect the overall speed and shape of the displacement front. Hence, the disagreement in the position of $S_{\mathrm{w}}$ as a function of time appears at the largest values of $S_{\mathrm{w}}$.

The influence of capillary pressure on relative permeability is reflected in the curvature of the two curves, greater curvature indicates that capillary pressure is larger and the radius of pores and throats is smaller. In Fig. 10, the water phase permeability rises slowly at first and then increases rapidly, while the oil phase permeability declines rapidly.

In Figs. 11 and 12, we see that the measured experimental curves are close to the history matching curves. The water-oil front arrived at almost the same position and the differential pressure curves are approximately consistent at

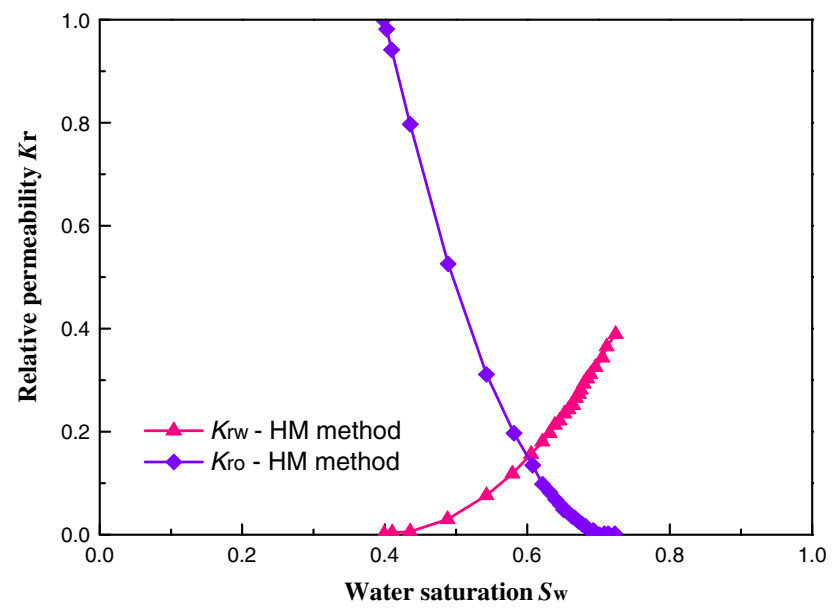

Fig. 10 The relative permeability curve derived from history matching method the same time. The influence of capillary end effects is eliminated in the calculation of saturation, since only the center section of core was scanned (both $1 \mathrm{~cm}$ of the core inlet and outlet end were not scanned) via X-ray CT technique to obtain water saturation profiles. Moreover, the problem of multiple solutions in history matching process is eliminated through simultaneous matching of the water saturation profiles data and the pressure differential data.

Mathematical calculating (MC) method

The mathematical model used for the interpretation of displacement data is based on the standard one-dimensional equation for two-phase (immiscible) flow in porous media. For the wetting phase (water), the Darcy velocity is given by the extended Darcy's law,

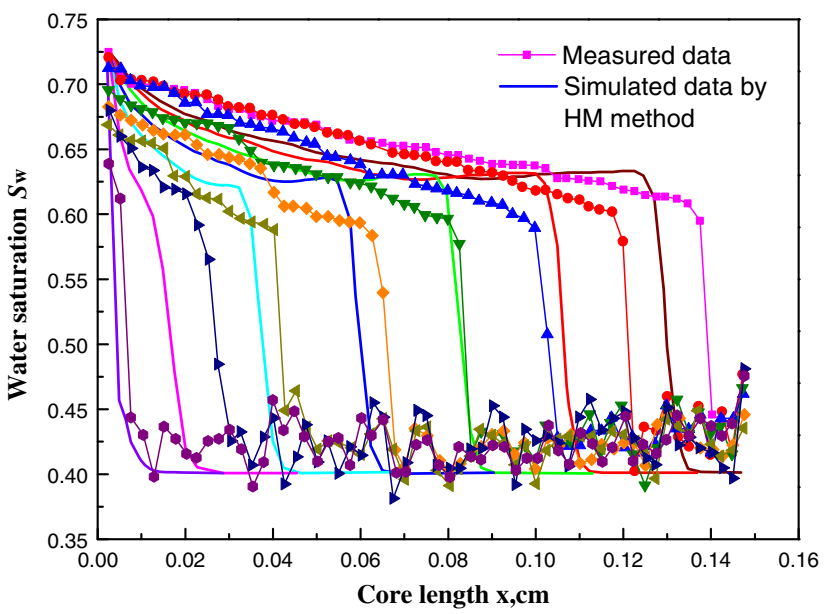

Fig. 11 The water saturation profiles obtained from simulated data and experimental data

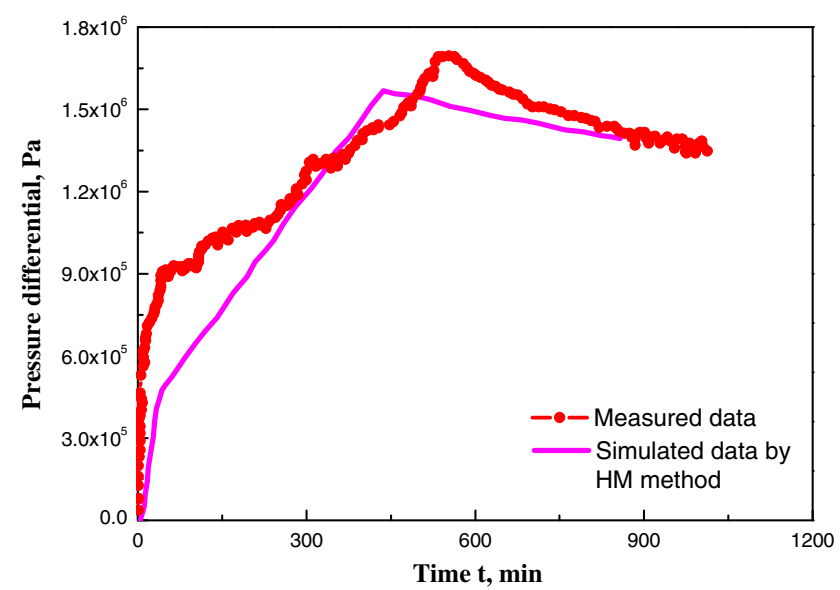

Fig. 12 The differential pressure obtained from simulated data and experimental data 
$u_{\mathrm{w}}=-\frac{k k_{\mathrm{rw}}}{\mu_{\mathrm{w}}} \frac{\partial p_{\mathrm{w}}}{\partial x}$

$\frac{\partial u_{\mathrm{w}}}{\partial x}=-\varphi \frac{\partial S_{\mathrm{w}}}{\partial t}$

$u_{w}-u_{z}=\int_{0}^{x}-\varphi \frac{\partial S_{\mathrm{w}}}{\partial t} \mathrm{~d} x$

$u_{z}-\int_{0}^{x} \varphi \frac{\partial S_{\mathrm{w}}}{\partial t} \mathrm{~d} x=-\frac{k k_{\mathrm{rw}}}{\mu_{\mathrm{w}}} \frac{\partial p_{\mathrm{w}}}{\partial x}$

As a result, the water relative permeability is obtained,

$k_{\mathrm{rw}}=-\mu_{\mathrm{w}}\left(u_{z}-\int_{0}^{x} \varphi \frac{\partial S_{\mathrm{w}}}{\partial t} \mathrm{~d} x\right) /\left(k \frac{\partial p_{\mathrm{w}}}{\partial x}\right)$

The pressure in the core,

$\Delta p_{\mathrm{w}}+\Delta p_{\mathrm{o}}-p_{\mathrm{c}}=p_{\mathrm{t}}$

where $\Delta p_{\mathrm{w}}$ is the pressure gradient of water phase, $\Delta p_{\mathrm{o}}$ is the pressure gradient of oil phase, $p_{\mathrm{c}}$ is the capillary pressure, $p_{\mathrm{t}}$ is the differential pressure at the inlet-outlet ends of the core.

Neglecting the pressure gradient of oil phase, we have the pressure gradient of water phase

$\Delta p_{\mathrm{w}}=p_{\mathrm{t}}+p_{\mathrm{c}}$

$\frac{\partial p_{\mathrm{w}}}{\partial x}=\frac{\partial p_{\mathrm{t}}}{\partial x}+\frac{\partial p_{\mathrm{c}}}{\partial x}$

Considering the dynamic capillary pressure (Eqs. 1, 2), then we get

$\frac{\partial p_{\mathrm{w}}}{\partial x}=\frac{\partial p_{\mathrm{t}}}{\partial x}+\frac{\partial p_{\mathrm{c}}^{\mathrm{e}}}{\partial S_{\mathrm{w}}} \frac{\partial S_{\mathrm{w}}}{\partial x}-\tau \frac{\partial}{\partial x}\left(\frac{\partial S_{\mathrm{w}}}{\partial t}\right)$

The relative permeability ratio calculated by fractional flow equation is used to obtain the oil relative permeability (Janos et al. 2001).

Firstly, the variation rate of water saturation at $x$ position is calculated using the numerical method based on the water saturation profiles obtained via X-ray CT scanning.

$\frac{\partial S_{\mathrm{w}}}{\partial t}=\frac{S_{\mathrm{w}}(x, t+\Delta t)-S_{\mathrm{w}}(x, t)}{\Delta t}$

Secondly, the water and oil flow velocity at $x$ position (the corresponding space node is $i$ ) is given according to the mass conservation equation of fluid, respectively:

$q_{o, i}=q_{o, i-1}-\frac{A\left(\mathrm{~S}_{\mathrm{w}}(x, t+\Delta t)-\mathrm{S}_{\mathrm{w}}(x, t)\right) \mathrm{d} x}{\Delta t}$

$q_{w, i}=q-q_{o, i}$

Then, we have the relative permeability ratio and the oil relative permeability can be obtained via numerical method, $\left(\frac{k_{\mathrm{ro}}}{k_{\mathrm{rw}}}\right)_{i}=\frac{q_{o, i} \mu_{\mathrm{o}}}{q_{w, i} \mu_{\mathrm{w}}}$

Finally, the calculated relative permeability curves are showed in Fig. 13. Through this method, the capillary end effect is avoided and the dynamic capillary pressure is considered.

\section{Comparison}

We validated the method embodied in Eqs. 26 and 34. The relative permeability curves obtained, using Eqs. 26 and 34 , were compared to the history matching curves in Fig. 14. The curves obtained are close to the history matching curves, with some scatter mainly related to the representation of a laboratory-scale experiment, indicating that both HM method and MC method can be applied in the calculation of two-phase relative permeability for ultra-low permeability reservoir. And the relative permeability curves of both methods have some similar features, e.g., the common seepage area of oil-water two phases is very narrow, the oil phase permeability decline rapidly, the water phase permeability rising slowly at first then increases rapidly.

However, we need also to see that the HM method is more accurate than the MC method, since the mathematical model is derived based on a series of assumptions. For the MC method, there are errors caused by assumptions, such as ignoring the pressure gradient of non-wetting phase and ignoring the effect of capillary pressure and gravity in the fractional flow equation, so that the influence of non-wetting phase pressure gradient and capillary pressure on relative permeabilities cannot be reflected accurately. While for the HM method, the water saturation profiles in waterflooding process are measured through X-ray CT

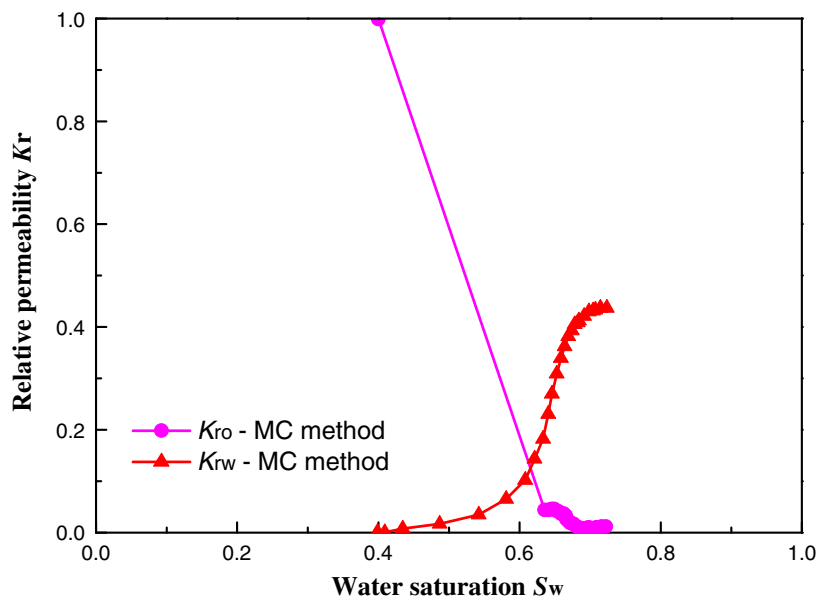

Fig. 13 The relative permeability curve derived from mathematic calculating method 


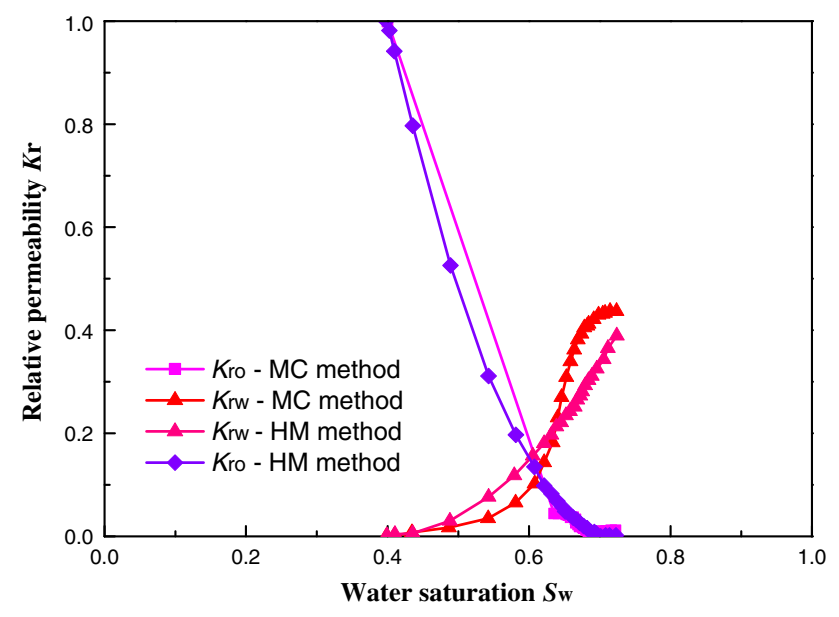

Fig. 14 The relative permeability curve derived from HM method and $\mathrm{MC}$ method

scanning. The simulated annealing algorithm can minimize the errors between simulated data and experimental measured data and the errors caused by assumptions are avoided at the most extent. Therefore, we think the HM method is more accurate than the MC method.

The advantages of the MC method lie in that the calculation of relative permeability is rapid considering the dynamic capillary pressure and is not influenced by the capillary end effect.

In Fig. 14, the MC method shows that the curve of water relative permeability becomes convex when $S_{\mathrm{w}}$ reaches residual oil saturation. The assumption that dynamic coefficient is a constant in the mathematic model may play a major role for the behavior in water relative permeability, since at the advancing oil-water front, $\tau_{\mathrm{s}}$ changes along with the variation of saturation. Hassanizadeh et al. (2002) suggested that dynamic capillary coefficient, $\tau_{\mathrm{s}}$, depends on saturation $\tau_{\mathrm{s}}=\tau_{\mathrm{s}}(S)$, and found that the value of $\tau_{\mathrm{s}}$ varies for different water saturation intervals. Another possible reason may be the calculation error caused by the model.

\section{Conclusions}

The calculated large coefficients $\tau_{\mathrm{s}}$ of dynamic capillary pressure demonstrate the obvious dynamic effect of capillary pressure in ultra-low permeability reservoir.

The JBN method (without considering capillary pressure) was used at first for calculating relative permeability curves using revised data, but the relative permeability curves are almost two intersecting straight lines. As the relative permeability is essential input for numerical simulation, neglecting the dynamic effect of capillary pressure while analyzing unsteady laboratory data ultimately leads to inaccurate assessment of multiphase flow properties. Thus, the JBN method did not apply.

HM method and MC method have been developed to determine dynamic relative permeability from the water saturation profile history obtained during displacement experiments considering the dynamic capillary pressure and eliminating the influence of capillary end effect via X-ray CT scanning. Through the HM method, the relative permeability curves obtained were used to simulate the water saturation profile and the differential pressure versus time. The simulated history reproduced the experimental behavior well. Notice that the shape and position of the displacement front are well reproduced. The MC method was compared with the HM method and the results were close, since they have the similar relative permeability features, suggesting the applicability for ultra-low permeability reservoirs. Importantly, the proposed methods allow measurement of relative permeability from a single experiment. Potentially, this represents a great time savings.

Acknowledgements This work is supported by the project (No. 51204193) sponsored by the Natural Sciences Foundation of China (NSFC).

Open Access This article is distributed under the terms of the Creative Commons Attribution License which permits any use, distribution, and reproduction in any medium, provided the original author(s) and the source are credited.

\section{References}

Akin S, Kovscek A R (1999) Imbibition studies of low-permeability porous media. In: SPE western regional meeting. doi:10.2118/ 54590-MS

Akin S, Schembre JM, Bhat SK, Kovscek AR (2000) Spontaneous imbibition characteristics of diatomite. J Petrol Sci Eng 25(3):149-165

Ali JK (1997) Developments in measurement and interpretation techniques in coreflood tests to determine relative permeabilities. In: Latin American and Caribbean petroleum engineering conference. doi:10.2118/39016-MS

Ayala REG, Aziz K (1993) Design and construction of an experiment for two-phase flow in fractured porous media (No.DOE/BC/ 14899-3; SUPRI-TR-95). Stanford Univ., CA (United States). Petroleum Research Inst

Bail PT, Marsden S S (1956) Saturation distribution in a linear system during oil displacement. In: Fall meeting of the petroleum branch of AIME. doi: 10.2118/695-G

Das DB, Mirzaei M (2012) Experimental measurement of dynamic effect in capillary pressure relationship for two-phase flow in weakly layered porous media. AIChE J. doi:10.1002/aic.13925

Das DB, Gauldie R, Mirzaei M (2007) Dynamic effects for two-phase flow in porous media: fluid property effects. AIChE J 53(10):2505-2520

Dixit AB, McDougall SR, Sorbie KS (1998) A pore-level investigation of relative permeability hysteresis in water-wet systems. SPE J 3(2):115-123 
Dixit A, Buckley J, McDougall S, Sorbie K (2000) Empirical measures of wettability in porous media and the relationship between them derived from pore-scale modelling. Transp Porous Media 40(1):27-54

Donaldson EC, Dean GW (1966) Two- and three-phase relative permeability studies. U.S.Bureau of Mines, Washington, DC

Fenwick DH, Blunt MJ (1998) Three-dimensional modeling of three phase imbibition and drainage. Adv Water Resour 21(2): $121-143$

Firoozabadi A, Aziz K (1988) Relative permeabilities from centrifuge data. In: SPE California regional meeting. doi:10.2118/15059MS

Hassanizadeh SM, Gray WG (1990) Mechanics and thermodynamics of multiphase flow in porous media including interphase boundaries. Adv Water Resour 13(4):169-186

Hassanizadeh SM, Celia MA, Dahle HK (2002) Dynamic effect in the capillary pressure-saturation relationship and its impacts on unsaturated flow. Vadose Zone J 1(1):38-57

Hirasaki GJ, Rohan JA, Dudley JW (1995) Interpretation of oil/water relative permeabilities from centrifuge displacement. SPE Adv Technol Ser 3(1):66-75

Honarpour M, Mahmood SM (1988) Relative-permeability measurements: an overview. J Petrol Technol 40(8):963-966

Hove AO, Ringen JK, Read PA (1987) Visualization of laboratory corefloods with the aid of computerized tomography of X-rays. SPE Reserv Eng 2(2):148-154

Huang DD, Honarpour MM (1998) Capillary end effects in coreflood calculations. J Petrol Sci Eng 19(1):103-117

Hughes RG, Brigham WE, Castanier LM (1996) CT imaging of two phase flow in fractured porous media. In: Proceedings of the $21 \mathrm{st}$ workshop on geothermal reservoir, engineering. Stanford University, Stanford, pp 22-24

Janos T, Tibor B, Peter S, Faruk C (2001) Direct determination of relative permeability from nonsteady-state constant pressure and rate displacements. In: SPE production and operations symposium. doi:10.2118/67318-MS

Kamath J, de Zabala EF, Boyer RE (1995) Water/oil relative permeability endpoints of intermediate-wet, low-permeability rocks. SPE Form Eval 10(1):4-10

Kirkpatrick S, Gelatt CD, Vecchi MP (1983) Optimization by simulated annealing. Science 220(4598):671-680

Lake LW (1989) Enhanced oil recovery, Chap. 3. Prentice Hall, Inc, Englewood Cliffs
Lenormand R, Touboul E, Zarcone C (1988) Numerical models and experiments on immiscible displacements in porous media. J Fluid Mech 189(1):165-187

Lü W, Liu Q, Zhang Z et al (2012) Measurement of three-phase relative permeabilities. Petrol Explor Dev 39(6):758-763

Mirzaei M, Das DB (2007) Dynamic effects in capillary pressuresaturations relationships for two-phase flow in 3D porous media: Implications of micro-heterogeneities. Chem Eng Sci 62(7): 1927-1947

Mitlin VS, Lawton BD, McLennan JD, Owen LB (1998) Improved estimation of relative permeability from displacement experiments. In: International petroleum conference and exhibition of Mexico. doi:10.2118/39830-MS

Qadeer S, Dehghani K, Ogbe DO, Ostermann RD (1988) Correcting oil/water relative permeability data for capillary end effect in displacement experiments. In: SPE California regional meeting. doi:10.2118/17423-MS

Stauffer F (1978) Time dependence of the relations between capillary pressure, water content and conductivity during drainage of porous media. In: IAHR symposium on scale effects in porous media, vol 29. Thessaloniki, Greece. pp 335-352

The Oil and Gas Professional Standards Compilation Group of People's Republic of China (2007) SY/T 5345-2007 the test method for relative permeability. Petroleum Industry Press, Beijing

Topp GC, Klute A, Peters DB (1967) Comparison of water contentpressure head data obtained by equilibrium, steady-state, and unsteady-state methods. Soil Sci Soc Am J 31(3):312-314

Ucan S, Civan F, Evans R D (1993) Simulated annealing for relative permeability and capillary pressure from unsteady-state nondarcy displacement. In: SPE Annual technical conference and exhibition. doi:10.2118/26670-MS

Vinegar HJ, Wellington SL (1987) Tomographic imaging of threephase flow experiments. Rev Sci Instrum 58(1):96-107

Wanna J (1982) Static and dynamic water content -pressure head relations of porous media. Colorado State University, Fort Collins

Wildenschild D, Hopmans JW, Simunek J (2001) Flow rate dependence of soil hydraulic characteristics. Soil Sci Soc Am J 65(1):35-48

Withjack EM (1988) Computed tomography for rock-property determination and fluid-flow visualization. SPE Form Eval 3(4):696-704 